\title{
Second Order Sliding Mode Observers for Fault Reconstruction in Power Networks
}

\author{
Chiara Mellucci, Prathyush P Menon, Christopher Edwards, Antonella Ferrara
}

August 30, 2017

\begin{abstract}
This paper proposes a 2-sliding mode observer to detect and reconstruct a certain class of load altering faults in a power network. The observer design is based on the recently proposed multivariable super-twisting structure. The IEEE benchmark power networks used to test the scheme are modelled as a semi-explicit class of differential algebraic equations (DAEs). For the purpose of developing the detection scheme, only the phase angles of the generators are measured, which represent a subset of the differential states of the DAEs. The objective is to estimate the differential states (the phase angles and frequencies of the generators), the algebraic states (the phase angles of the load bus tensions) and to reconstruct a class of load altering faults affecting the network. The proposed observer is assessed in simulation on two IEEE benchmarks: the 9-bus and 14-bus networks, so as to verify its capability to correctly estimate the differential and algebraic states of the network in spite of its complexity and uncertainty. Moreover, the capability of the proposed scheme to detect the presence of a load altering fault, to exactly identify its position in the network, and to precisely reconstruct the shape of the fault itself is shown and discussed.
\end{abstract}

\section{Introduction}

Infrastructures including power grids, water distribution networks and transport systems play an important role in the $21^{\text {st }}$ century and are becoming increasingly susceptible to disruptive attacks. In a power network, a fault in one component or a deliberate malicious action (such as unregulated absorption of power) could compromise the safe operation of the overall system. Such an event must be quickly identified, so that action can be taken to mitigate the problem. In this paper power networks will be considered, although the ideas which will be presented could be exploited in other applications. In particular, the faults considered in this paper represent a variation of the power consumption at the load buses. This can arise in two different ways: (i) as an unplanned increase in the power demand of a registered utility or as a load decrease due to an induction motor detachment; (ii) as a result of a utility consuming more than the allowed amount of power or injecting power into the network illegally. These actions affect the performance of the network, and therefore it is important to detect their presence in order to avoid instability in the network itself.

In this paper, the network model comprises a differential component (the linearised swing dynamics associated with the generators) and an algebraic component, representing the DC power flow equations. The resulting model is a set of semi-explicit differential algebraic equations (DAEs): that is a descriptor system [1]. Many different paradigms have been explored with the aim of creating a compact model for a power network; formulations similar to the one exploited in this paper can be found, for example, in [2], [3] and [4].

Different observer paradigms for DAE systems can be found in the literature. Unknown input observers have been used in [5] and [1] for linear descriptor systems in which the algebraic variables have been treated as unknown inputs. A sliding mode observer for a descriptor system in which the inputs do not enter the algebraic equations has been proposed in [6]; the observer design is based on the principle of singularly perturbed sliding manifolds. A centralized Luenberger observer, designed through the graphical procedure described in [7], and an $\mathcal{H}_{\infty}$ observer, based on the ideas in [8], have been designed in [3] for large power networks modelled as DAE systems. A class of sliding mode observers for descriptor systems is proposed and analysed in [9] and [10] but, as highlighted in the following, these schemes are not suitable for the DAE model of the power network when only phase angle measurements are available. Recently in [1], necessary and sufficient conditions for the existence of a Luenberger observer for an arbitrary order DAE were given, exploiting the concept of impulse observability. This paper considers a 2-sliding mode observer exploiting super-twisting structures [11], [12].

The use of state estimators for the detection of faults or exogenous signals is common in power network applications. In [13] a Kalman filter is used to estimate the state variables of a smart grid model, in order to detect the presence of external attacks, such as Denial of Service. In [14], a hybrid observer is used to detect 
faults occurring in a class of switched linear systems, used to model power converters. In [15] an extended state observer is used to estimate the load power, modelled as an external disturbance, of a grid connected power converter. The scale of the problem considered in these two applications is different, as the focus is on a single component of the power network, but the underlying strategy is the same.

The main contribution of this paper is the design of a multivariable super-twisting sliding mode observer for a class of semi-explicit DAE systems, used to model power networks. The proposed scheme is applied to the 9 and 14 bus IEEE benchmark models [16], to highlight its adaptability. A reduced measurement set is considered, assuming only the phase angles of the generators are measured. These measurements are shown to be sufficient for the estimation of the entire state of the DAE system (including both the differential and algebraic states) even in the presence of model parameter uncertainty. From these available measurements, moreover, the detection, identification and reconstruction of exogenous signals, representing faults, is achieved. The present scheme is inspired by the observer design proposed (for non DAE systems) in [17].

\section{Problem Definition and System Description}

The aim is to design an observer able to estimate the complete state vector of the DAE system representing the power network, and to detect the presence and reconstruct the 'shape' of load altering faults. Load altering faults can be interpreted as the unregulated consumption or production of power: they were originally defined in [4], and later studied in [18]. In this paper it is assumed that only a single failure/attack can occur at a particular time, i.e., simultaneous different faults are precluded. Although this is a restriction, it is in line with [19].

A network composed of $n_{g}$ generators and $n_{l}$ load buses is considered. The $i$-th generator is modelled through the swing dynamics [3], which is the differential equation representing Newton's second law expressed in rotational form. The swing dynamics is given by:

$$
m_{i} \ddot{\delta}_{i}+d_{i} \dot{\delta}_{i}-P_{g i}=-\sum_{j \in N_{i}} P_{i j}
$$

where $\delta_{i}$ is the $i^{t h}$ generator phase angle and $\dot{\delta}_{i}$ is its voltage frequency, $m_{i}$ and $d_{i}$ are the coefficients of inertia and damping, $P_{g i}$ is the mechanical input power at the $i^{t h}$ generator and $P_{i j}$ is the flow of active electrical power between node $i$ and its neighbouring nodes $j \in N_{i}$ [3]. The neighbourhood set $N_{i}$ includes the indices of the buses directly linked to the $i^{\text {th }}$ node through a line; consequently the topology of the network is embedded in the dynamics.

A static model has been chosen for the load buses, which have been represented as a constant power consumption $P_{l_{i}}$. Applying the principle of conservation of energy at each load bus, the flow of active/real and reactive/imaginary power is described through:

$$
\begin{aligned}
\sum_{j \in N_{i}} P_{i j}(t)= & \sum_{j \in N_{i}}\left|V_{i}\right|\left|V_{j}\right|\left(G_{i j} \cos \left(\vartheta_{i}-\vartheta_{j}\right)+\right. \\
& \left.+B_{i j} \sin \left(\vartheta_{i}-\vartheta_{j}\right)\right)+\left|V_{i}\right|^{2} G_{i i} \\
\sum_{j \in N_{i}} Q_{i j}(t)= & \sum_{j \in N_{i}}\left|V_{i}\right|\left|V_{j}\right|\left[G_{i j} \sin \left(\vartheta_{i}-\vartheta_{j}\right)-\right. \\
& \left.-B_{i j} \cos \left(\vartheta_{i}-\vartheta_{j}\right)\right]-\left|V_{i}\right|^{2} B_{i i}
\end{aligned}
$$

where $\vartheta_{i}$ is the phase angle of the $i^{\text {th }}$ load bus tension $V_{i}$ [4]. Each line, connecting the $i^{\text {th }}$ and $j^{\text {th }}$ nodes, is characterized by a value of conductance $G_{i j}$ and susceptance $B_{i j}$. In addition, $G_{i i}=\sum_{j \in N_{i}}-G_{i j}$ and $B_{i i}=\sum_{j \in N_{i}}-B_{i j}$. The quantities in (2) are expressed in p.u.: this refers to the ratio between the actual value of the variable and a base or reference value. Usually, a base power and a base voltage are chosen, and reference values for the other electrical quantities are derived. A set of standard assumptions, similar to those in [7], [19], [20], [21], is introduced: (i) the value of the conductance $G_{i j}$ of each line can be neglected with respect to the susceptance $B_{i j}$; (ii) small angle approximations can be applied throughout the network; (iii) the voltages, expressed in p.u., are close to 1 p.u. and can be approximated by this value. A detailed discussion of these hypotheses can be found in [2].

Since $G_{i j}$ is negligible $\forall i, j, V_{i}=V_{j}=1 p . u . \forall i, j$ and $B_{i i}=\sum_{j \in N_{i}}-B_{i j}$, the right hand side of (3) is approximately zero. Consequently, the reactive power flow is neglected and the active power flow can be 


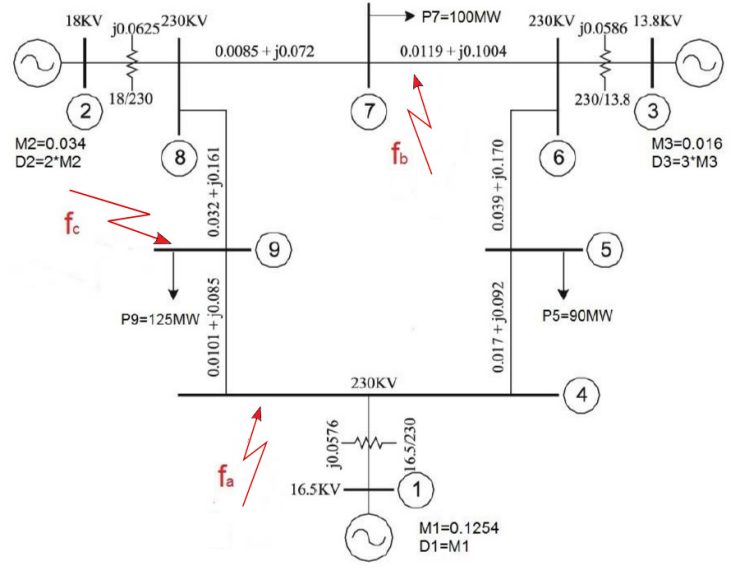

(a) IEEE - 9 Bus Benchmark

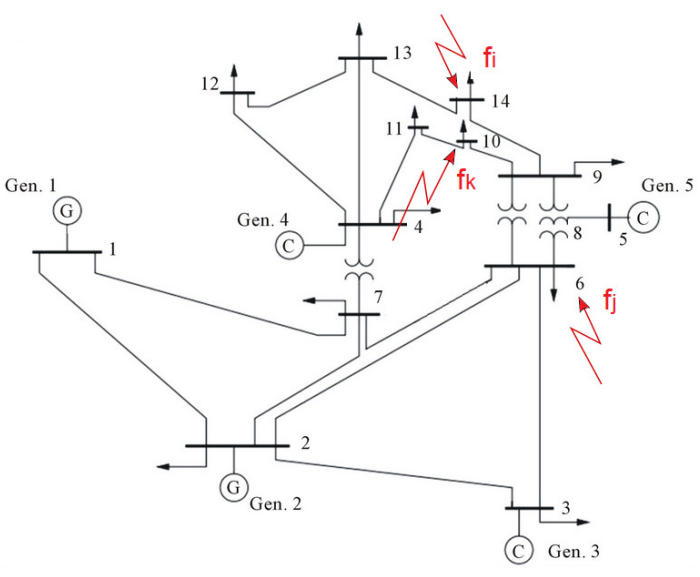

(b) IEEE - 14 Bus Benchmark

Figure 1: IEEE benchmarks and introduced faults

described through the following linearised small angle approximation:

$$
P_{l_{i}}=\sum_{j \in N_{i}} P_{i j}(t)=\sum_{j \in N_{i}}\left(B_{i j}\left(\vartheta_{i}-\vartheta_{j}\right)\right)=\sum_{j=1}^{N} \mathcal{L}_{i j} \vartheta_{j}
$$

where $N=n_{g}+n_{l}$ is the total number of buses in the network. The matrix $\mathcal{L} \in \mathbb{R}^{N \times N}$ is the weighted graph Laplacian in which the topology of the network is embedded. The weights used to define the Laplacian $\mathcal{L}$ are the susceptances of the network lines. For later use in the paper, the following partition of the matrix $\mathcal{L}$ is introduced:

$$
\mathcal{L}=\left[\begin{array}{c:c}
\mathcal{L}_{g g} & \mathcal{L}_{g l} \\
\hdashline \mathcal{L}_{l g} & \mathcal{L}_{l l}^{-}
\end{array}\right]
$$

where $\mathcal{L}_{l l} \in \mathbb{R}^{n_{l} \times n_{l}}$ and the other matrices are partitioned accordingly. A set of algebraic state variables results from the active power flow equations in (4): these are the phase angles of the load bus tensions $\left(\vartheta_{i}\right)$.

The state vector $x(t) \in \mathbb{R}^{n}$, with $n=2 n_{g}+n_{l}$ is given by

$$
x(t):=\left[x_{d}(t)^{T} \mid x_{a}(t)^{T}\right]^{T}=\left[\begin{array}{ll}
\delta(t)^{T} & \omega(t)^{T} \mid \vartheta(t)^{T}
\end{array}\right]^{T}
$$

where $x_{d}(t)=\left[\begin{array}{ll}\delta(t)^{T} & \omega(t)^{T}\end{array}\right] \in \mathbb{R}^{2 n_{g}}$ is the differential state vector comprising the generator phase angles and frequencies, while $x_{a}(t)=\vartheta(t) \in \mathbb{R}^{n_{l}}$ is the algebraic state vector of the load bus tensions phase angles. The resultant linearised dynamics encapsulating (1) and (4) can be written in the following compact form:

$$
\begin{aligned}
E \dot{x}(t) & =A x(t)+B u(t)+d f(t), \\
y(t) & =C x(t) .
\end{aligned}
$$

The control input in (6) is

$$
u(t)=\left[\begin{array}{ll}
P_{g}^{T} & P_{l}^{T}
\end{array}\right]^{T} \in \mathbb{R}^{n_{g}+n_{l}}
$$

where $P_{g}$ and $P_{l}$ represent the input power at the $n_{g}$ generator buses and the consumed power at the $n_{l}$ load buses respectively.

The matrix $E \in \mathbb{R}^{n \times n}$ appearing in the left hand side of (6) is given by:

$$
E=\left[\begin{array}{c:c}
E_{d} & 0 \\
\hdashline 0 & 0
\end{array}\right]=\left[\begin{array}{cc:c}
I_{n_{g}} & 0 & 0 \\
0 & \mathcal{M}_{g} & 0 \\
\hdashline 0 & 0 & 0
\end{array}\right],
$$

where $E_{d}:=\operatorname{Diag}\left(I_{n_{g}}, \mathcal{M}_{g}\right) \in \mathbb{R}^{n_{d} \times n_{d}}$ is full rank, with $n_{d}=2 n_{g}$, and $\mathcal{M}_{g}=\operatorname{Diag}\left(m_{1}, \ldots m_{n_{g}}\right) \in \mathbb{R}^{n_{g} \times n_{g}}$ is the diagonal matrix of generator inertia coefficients. Clearly $\mathcal{R} a n k(E)=2 n_{g}<n$, and, as a consequence, $E$ is rank deficient. The DAE system is in semi-explicit form [22], as the algebraic states appear linearly in (6). In (6) the system matrix

$$
A=\left[\begin{array}{c:c}
A_{d} & A_{d a} \\
\hdashline A_{a d} & A_{a}^{-}
\end{array}\right]=\left[\begin{array}{cc:c}
0 & I_{n_{g}} & 0 \\
-\mathcal{L}_{g g} & -\mathcal{D}_{g} & -\mathcal{L}_{g l} \\
\hdashline-\overline{\mathcal{L}}_{l g} & 0 & -\mathcal{L}_{l l}
\end{array}\right],
$$


where $\mathcal{D}_{g}=\operatorname{Diag}\left(d_{1}, \ldots d_{n_{g}}\right)$ is the diagonal matrix of generator damping coefficients. The input and the fault distribution matrices are as follows:

$$
\begin{aligned}
B=\left[B_{d}^{T} \mid B_{a}^{T}\right]^{T} & =\left[\begin{array}{c|c}
0_{n_{g}} & 0_{n_{g} \times n_{l}} \\
I_{n_{g_{-}}} & 0_{n_{g} \times n_{l}} \\
\hdashline 0_{n_{l} \times n_{g}} & \underline{I}_{n_{l}}
\end{array}\right], \\
d & =\left[\begin{array}{c}
0 \\
d_{a}
\end{array}\right]
\end{aligned}
$$

where $d_{a}$ is a vector from the standard basis for $\mathbb{R}^{n_{l}}$, i.e., of the form $\operatorname{Col}(0, \ldots, 1, \ldots, 0)$ where the position of the unitary element indicates the particular load bus affected by the fault. The structure in (11) reflects the assumption that the faults are in the algebraic equations. Finally in (7)

$$
C=\left[\begin{array}{ll}
C_{d} & 0_{n_{l}}
\end{array}\right]=\left[\begin{array}{ll}
I_{n_{g}} & 0_{n_{g}+n_{l}}
\end{array}\right],
$$

which captures the fact that the differential states $\delta(t)$ are the only measured outputs; this strongly differentiates the present work from $[18,19]$.

The descriptor model (6) - (7) is an approximation of the real system behaviour. Model uncertainty can be captured by allowing the system matrices to vary inside a certain range [4]. In particular, uncertainty in the generator parameters would result in a variation of the diagonal matrices $\mathcal{M}_{g}$ and $\mathcal{D}_{g}$, while uncertainty on the line parameters would result in a perturbation term affecting the corresponding entries of the matrix $A$ [7]. In what follows the initial conditions $(x(0))$ are assumed to satisfy the algebraic constraints, which are related to the physics of the problem. The signal $f(t)$ appearing in (6) is an unknown exogenous signal which models a load altering fault acting in one of the algebraic channels associated with the load buses. In (6), the vector $d$ is linked to the channel in which the fault occurs, and is unknown. It is clear from (4) that changing the phase angle of a load bus tension $(\vartheta)$ modifies the power flows in all the lines passing through that node. Consequently, the entire network can be affected by a single fault.

Lemma 1 A necessary and sufficient condition for the triplet $(C, E, A)$ in (6) - (7) to be impulse observable is that the matrix $A_{a}$ from (9) satisfies $\operatorname{det}\left(A_{a}\right) \neq 0$.

Proof: It is shown in [1] that the triplet $(C, E, A)$ is impulse observable if and only if:

$$
\mathcal{R} a n k \underbrace{\left(\left[\begin{array}{cc}
E & A \\
0 & C \\
0 & E
\end{array}\right]\right)}_{\Theta}=\mathcal{R} \operatorname{Rank}(E)+n .
$$

where $n=2 n_{g}+n_{l}$ is the dimension of the state vector. Substituting for $E, A$ and $C$ from (8), (9) and (12):

$$
\Theta=\left[\begin{array}{cc:cc}
E_{d} & 0 & A_{d} & A_{d a} \\
0 & 0 & A_{a d} & A_{a} \\
\hdashline 0 & 0 & \bar{C}_{d} & 0 \\
\hdashline 0 & 0 & \bar{E}_{d} & 0 \\
0 & 0 & 0 & 0
\end{array}\right]
$$

where $C_{d}=\left[\begin{array}{ll}I_{n_{g}} & 0_{n_{g}}\end{array}\right]$. From (14), it can be seen that:

$$
\mathcal{R} a n k(\Theta)=\mathcal{R} a n k\left(E_{d}\right)+\mathcal{R} a n k\left(\left[\begin{array}{cc}
A_{a d} & A_{a} \\
C_{d} & 0 \\
E_{d} & 0
\end{array}\right]\right)
$$

Since $\operatorname{Rank}(E)=\mathcal{R} a n k\left(E_{d}\right)$ and $\operatorname{det}\left(E_{d}\right) \neq 0$, from (15):

$$
\mathcal{R} a n k(\Theta)=\mathcal{R} a n k(E)+\mathcal{R} a n k\left(\left[\begin{array}{cc}
0 & A_{a} \\
E_{d} & 0
\end{array}\right]\right)
$$

Moreover:

$$
\begin{aligned}
\operatorname{Rank}\left(\left[\begin{array}{cc}
0 & A_{a} \\
E_{d} & 0
\end{array}\right]\right)=n & \Leftrightarrow \operatorname{Rank}\left(A_{a}\right)=n_{l} \\
& \Leftrightarrow \operatorname{det}\left(A_{a}\right) \neq 0
\end{aligned}
$$

Hence necessary and sufficient conditions for (13) to be satisfied is that $\operatorname{det}\left(A_{a}\right) \neq 0$. 


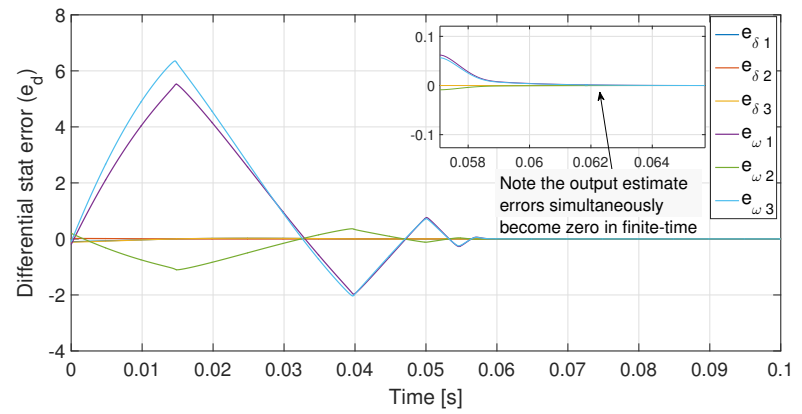

(a) Error in differential state estimate $e_{d}=\left[\begin{array}{ll}e_{\delta} & e_{\omega}\end{array}\right]^{T}$

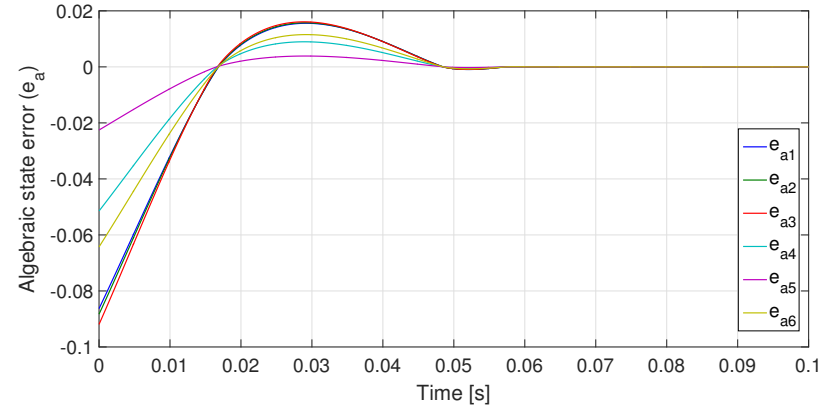

(b) Error in algebraic state estimate $e_{a}$

Figure 2: Convergence of the state estimations - 9-bus scenario

Remark 1 The condition $\operatorname{det}\left(A_{a}\right) \neq 0$ is of course a restriction; however this assumption has been previously made in [18]. From (9), $A_{a}=-\mathcal{L}_{l l}$ and in power networks, this Laplacian sub-matrix is invertible [18]. Consequently $(A, E, C)$ is impulse observable.

Making use of the hypotheses and the structure in (8) - (12), the DAE in (6) and (7) can be rewritten as:

$$
\begin{aligned}
\dot{x}_{d}(t) & =E_{d}^{-1}\left(A_{d} x_{d}(t)+A_{d a} x_{a}(t)+P_{g}(t)\right), \\
0 & =A_{a d} x_{d}(t)+A_{a} x_{a}(t)+P_{l}(t)+d_{a} f(t), \\
y(t) & =C_{d} x_{d}(t) .
\end{aligned}
$$

From the arguments above, $\operatorname{det}\left(A_{a}\right) \neq 0$ and the algebraic states can be expressed as a function of the differential states, the control inputs and the exogenous signals. Specifically, from (18):

$$
x_{a}(t)=A_{a}^{-1}\left(A_{a d} x_{d}(t)+B_{a} u(t)+d_{a} f(t)\right) .
$$

The system in (17), (19) and (20) is a 'collapsed model'. This reduced order system, in power network applications, is said to be the Kron-reduction of the descriptor power system model [18]. This is a system of index ' 1 ' since the graph Laplacian matrix that embodies the topology of the network is irreducible because of the assumed connectivity.

\section{Multivariable super-twisting Sliding Mode Observer}

The proposed observer for the DAE in (6) is:

$$
E \dot{z}(t)=A z(t)+B u(t)+K e_{y}(t)+\Phi\left(e_{y}(t)\right),
$$

where $e_{y}(t):=C(x(t)-z(t))$ is the output estimation error and $z(t)$ is the state estimate. The injection term

$$
\Phi\left(e_{y}(t)\right):=\operatorname{Col}\left(\Phi_{d}\left(e_{y}(t)\right), 0\right)
$$

is a function of the output estimation error and $\Phi_{d}\left(e_{y}(t)\right) \in \mathbb{R}^{n_{d}}$. The observer gain matrix is assumed to be of the form $K:=\mathcal{C}$ ol $\left(K_{d}, 0\right)$ where $K_{d} \in \mathbb{R}^{n_{d} \times n_{g}}$ is to be designed.

If the state estimation error for the DAEs is defined as $e(t):=x(t)-z(t)=\left[e_{d}(t)^{T}, e_{a}(t)^{T}\right]^{T}$, then subtracting (21) from (6), the state estimation error dynamics satisfies

$$
E \dot{e}(t)=A e(t)+d f(t)-K e_{y}(t)-\Phi\left(e_{y}(t)\right) .
$$

The dynamics in (22) can be partitioned into differential and algebraic error states so that

$$
\begin{aligned}
E_{d} \dot{e}_{d}(t) & =A_{d} e_{d}(t)+A_{d a} e_{a}(t)-K_{d} e_{y}(t)-\Phi_{d}\left(e_{y}(t)\right) \\
0 & =A_{a d} e_{d}(t)+A_{a} e_{a}(t)+d_{a} f(t) .
\end{aligned}
$$

Since $\operatorname{det}\left(A_{a}\right) \neq 0$, the error in the algebraic variable estimates, $e_{a}(t)$, can be evaluated, from (24), as:

$$
e_{a}(t)=-A_{a}^{-1}\left(A_{a d} e_{d}(t)+d_{a} f(t)\right) .
$$




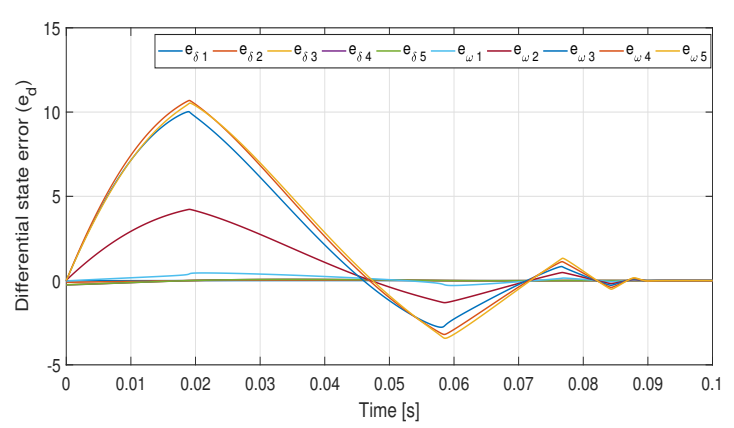

(a) Error in differential state estimate $e_{d}=\left[\begin{array}{ll}e_{\delta} & e_{\omega}\end{array}\right]^{T}$

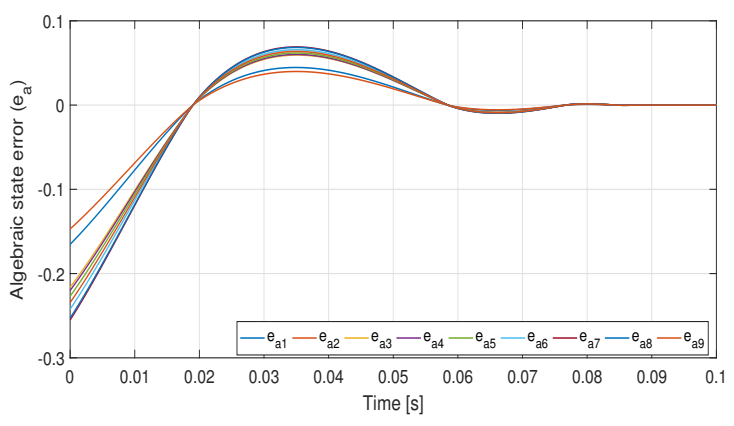

(b) Error in algebraic state estimate $e_{a}$

Figure 3: Convergence of the state estimations - 14-bus scenario in presence of uncertainty in the plant

Substituting (25) in (23) yields

$$
\begin{aligned}
E_{d} \dot{e}_{d}(t)= & \left(A_{d}-A_{d a} A_{a}^{-1} A_{a d}\right) e_{d}(t)-K_{d} e_{y}(t)- \\
& -\Phi_{d}\left(e_{y}(t)\right)-\left(A_{d a} A_{a}^{-1} d_{a}\right) f(t) .
\end{aligned}
$$

Let $e_{d}:=\left(e_{\delta}, e_{\omega}\right)$ where $e_{\delta} \in \mathbb{R}^{n_{g}}$ and $e_{\omega} \in \mathbb{R}^{n_{g}}$ and define

$$
K_{d}:=\left[\begin{array}{c}
-\mathcal{M}_{g}^{-1} \mathcal{D}_{g}+k_{2} I_{n_{g}} \\
k_{4} \mathcal{M}_{g}-\mathcal{L}_{g g}+\mathcal{L}_{g l} \mathcal{L}_{l l}^{-1} \mathcal{L}_{l g}+\mathcal{D}_{g} \mathcal{M}_{g}^{-1} \mathcal{D}_{g}-k_{2} \mathcal{D}_{g}
\end{array}\right]
$$

and

$$
\Phi_{d}\left(e_{y}(t)\right):=\left[\begin{array}{c}
k_{1} \frac{e_{y}(t)}{\left\|e_{y}(t)\right\|^{\frac{1}{2}}} \\
-k_{1} \mathcal{D}_{g} \frac{e_{y}(t)}{\left\|e_{y}(t)\right\|^{\frac{1}{2}}}+k_{3} \mathcal{M}_{g} \frac{e_{y}(t)}{\left\|e_{y}(t)\right\|}
\end{array}\right]
$$

for $e_{y}(t) \neq 0$. Define $\bar{f}(t)=\mathcal{M}_{g}^{-1} \mathcal{L}_{g l} \mathcal{L}_{l l}^{-1} d_{a} f(t)$, then from (8)-(12), equation (26) can be rewritten as:

$$
\begin{aligned}
\dot{e}_{\delta}(t) & =e_{\omega}(t)+\mathcal{M}_{g}^{-1} \mathcal{D}_{g} e_{\delta}(t)-k_{2} e_{\delta}(t)-k_{1} \frac{e_{\delta}(t)}{\left\|e_{\delta}(t)\right\|^{\frac{1}{2}}} \\
\dot{e}_{\omega}(t) & =\left(k_{2} \mathcal{M}_{g}^{-1} \mathcal{D}_{g}-\mathcal{M}_{g}^{-1} \mathcal{D}_{g} \mathcal{M}_{g}^{-1} \mathcal{D}_{g}-k_{4} I\right) e_{\delta}(t)-\bar{f}(t)- \\
& -\mathcal{M}_{g}^{-1} \mathcal{D}_{g} e_{\omega}(t)+k_{1} \mathcal{M}_{g}^{-1} \mathcal{D}_{g} \frac{e_{y}(t)}{\left\|e_{y}(t)\right\|^{\frac{1}{2}}}-k_{3} \frac{e_{\delta}(t)}{\left\|e_{\delta}(t)\right\|}
\end{aligned}
$$

where $k_{i}>0$, for $i=1, \ldots, 4$. From the structure of $C$ in $(12), e_{y}(t) \equiv e_{\delta}(t)$. Let $\tilde{e}_{\omega}=e_{\omega}+\mathcal{M}_{g}^{-1} \mathcal{D}_{g} e_{\delta}(t)$, then the error dynamics can be written as:

$$
\begin{aligned}
& \dot{e}_{\delta}(t)=\tilde{e}_{\omega}(t)-k_{2} e_{\delta}(t)-k_{1} \frac{e_{\delta}(t)}{\left\|e_{\delta}(t)\right\|^{\frac{1}{2}}} \\
& \dot{\tilde{e}}_{\omega}(t)=-k_{4} e_{\delta}(t)-k_{3} \frac{e_{\delta}}{\left\|e_{\delta}\right\|}-\bar{f}(t)
\end{aligned}
$$

The following assumption is imposed [23].

Assumption $1 \bar{f}(t)$ is bounded by a known constant $\eta$.

The introduction of Assumption 1 is possible as the signal $\bar{f}(t)$ only depends on the fault signal $f(t)$ and not on $e_{\delta}$ or $e_{\omega}$.

Theorem 1 For the error dynamics in (31) - (32), there exist positive scalar gains, $k_{1}, k_{2}, k_{3}$ and $k_{4}$, depending on $\eta$ such that $e_{\delta}(t)=\dot{e}_{\delta}(t) \equiv 0$ in finite time and the origin is invariant for all subsequent time.

Proof: A sketch of the proof will be given. The representation in (31)-(32) is as special case of the structure in [17]. The work in [17] proposes a set of inequalities for $k_{1}, k_{2}, k_{3}$ and $k_{4}$ in terms of $\eta$. These inequalities guarantee that the function:

$$
V\left(e_{\delta}, e_{\omega}\right)=2 k_{3}\left\|e_{\delta}\right\|+k_{4} e_{\delta}^{T} e_{\delta}+\frac{1}{2} e_{\omega}^{T} e_{\omega}+\frac{1}{2} \xi^{T} \xi
$$


Table 1: Observer's design parameters

\begin{tabular}{|c|c|c|c|c|}
\hline$\eta$ & $k_{1}$ & $k_{2}$ & $k_{3}$ & $k_{4}$ \\
\hline 100 & 15 & 25 & 390 & 2625 \\
\hline
\end{tabular}

where

$$
\xi:=k_{1} \frac{e_{\delta}}{\left\|e_{\delta}\right\|^{1 / 2}}+k_{2} e_{\delta}-e_{\omega}
$$

is a Lyapunov function for (31)-(32) which becomes identically equal to zero in finite time and remains at zero [17]. From Lyapunov theory, it can be argued that $e_{\delta}(t)=\dot{e}_{\delta}(t) \equiv 0$ in finite time and a 2-SM takes place.

Remark 2 In the absence of a good estimation of $\eta$, the possibility of designing an adaptive super-twisting sliding mode observer could be considered [24], [25].

Remark 3 A sliding mode observer for descriptor systems of the form (6)-(7) has been proposed in [9], [10], assuming $\mathcal{R}$ ank $\left(\left[\begin{array}{cc}E^{T} & C^{T}\end{array}\right]\right)=n$. As a consequence, the application of these schemes to the current problem is not possible since, from (8) and (12):

$$
\mathcal{R a n k}\left(\left[\begin{array}{ll}
E^{T} & C^{T}
\end{array}\right]\right)=2 n_{g}=n-n_{l}<n
$$

\section{Fault Detection and Reconstruction}

During the sliding motion, $e_{\delta}=0$ and $\dot{e}_{\delta}=0$ and the unit vector component in (30) must on average take a value to compensate for $\mathcal{M}_{g}^{-1} \mathcal{L}_{g l} \mathcal{L}_{l l}^{-1} d_{a} f(t)$. Writing the equivalent output error injection term as $\nu_{e q}(t)$, equation (30), during sliding, takes the form:

$$
\nu_{e q}(t)+\underbrace{\mathcal{M}_{g}^{-1} \mathcal{L}_{g l} \mathcal{L}_{l l}^{-1}}_{\Psi} d_{a} f(t)=0
$$

A good approximation of $\nu_{e q}(t) \in \mathbb{R}^{n_{g}}$ can be obtained in real time [26]. In (35), $\Psi \in \mathbb{R}^{n_{g} \times n_{l}}$ is known, but both $d_{a}$ and $f(t)$ are unknown. When $\Psi$ is a square matrix and hence (potentially) invertible, it is straightforward to estimate the unknown failure $d_{a} f(t)$ by multiplying both sides of (35) by $\Psi^{-1}$. However here $\Psi$ is not square, and hence not invertible. Consequently, the load altering fault is not easily estimated through a linear combination of the equivalent injection signals $\nu_{e q}(t)$. However, an alternative estimation method can be introduced: this three-stage method exploits the fault signature vector $d_{a}$. The first stage of the method is fault detection, to identify the presence of the fault. The norm of the equivalent injection signal from (35) is $\left\|\nu_{e q}\right\|=\left\|\Psi d_{a}\right\||f(t)|$, and since $d_{a}$ is a vector from the standard basis of $\mathbb{R}^{n_{l}}, \Psi d_{a}=\Psi_{i} \in \mathbb{R}^{n_{g}}$ where $\Psi_{i}$ is a column of $\Psi$. If $\Psi_{i} \neq 0$, then $\left\|\nu_{e q}\right\|$ can be used as an indicator of the presence of a fault. Thus, fault detection is achieved testing the condition:

$$
\left\|\nu_{e q}\right\| \geq \epsilon
$$

where $\epsilon$ is a positive design threshold used to trigger an alarm. The detection of the fault is followed by a) the identification of the channel affected by the fault; and b) the reconstruction of the fault's shape.

The fault identification procedure is based on directional characterization. A fault/attack on the $i^{\text {th }}$ load bus is associated with the $i^{\text {th }}$ column of $\Psi$, that is $\Psi_{i}$. As a consequence, during this stage the aim is to identify the column of $\Psi$ that best matches the vector $\nu_{e q}(t)$ in terms of its direction cosine [27]. (Recall that a unitary cosine value indicates perfect alignment between two vectors). Specifically, the fault channel is identified as

\begin{tabular}{|c|c|c|c|c|c|c|c|c|c|c|c|c|}
\hline & \multicolumn{4}{|c|}{ Fault 1} & \multicolumn{4}{|c|}{ Fault 2} & \multicolumn{4}{|c|}{ Fault 3} \\
\hline & Fault & Bus & Interval (s) & Signal & Fault & Bus & Interval (s) & Signal & Fault & Bus & Interval (s) & Signal \\
\hline 9 - bus & $f_{a}(t)$ & 4 & $2-7$ & $\begin{array}{l}\text { Sine } \\
\text { Amplitude: } 2 \text { p.u. } \\
\text { Frequency: } 1.5 \mathrm{~Hz}\end{array}$ & $f_{b}(t)$ & 7 & $9-14$ & $\begin{array}{c}\text { Ramp } \\
\text { Slope:-0.4 }\end{array}$ & $f_{c}(t)$ & 9 & $16-21$ & $\begin{array}{c}\text { Step } \\
\text { Amplitude: } 1.5 \text { p.u. }\end{array}$ \\
\hline 14 - bus & $f_{i}(t)$ & 14 & $2-6$ & $\begin{array}{c}\text { Step } \\
\text { Amplitude: }-1.2 \text { p.u. }\end{array}$ & $f_{j}(t)$ & 6 & $8-12$ & $\begin{array}{l}\text { Sine } \\
\text { Amplitude: } 2 \text { p.u. } \\
\text { Frequency: } 1.5 \mathrm{~Hz}\end{array}$ & $f_{k}(t)$ & 10 & $14-18$ & $\begin{array}{l}\text { Ramp } \\
\text { Slope: } 0.3\end{array}$ \\
\hline
\end{tabular}

$$
j:=\arg \max _{i=1 \ldots n_{l}}\left(\frac{\left|\nu_{e q}^{T} \Psi_{i}\right|}{\left\|\nu_{e q}\right\|\left\|\Psi_{i}\right\|}\right)
$$

Table 2: Characterization of the faults introduced in the 9 and 14 buses scenarios 


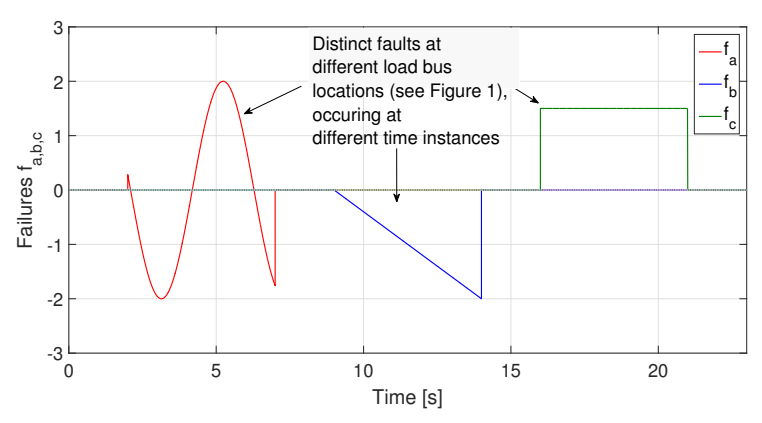

(a) Fault signals $f_{a}, f_{b}$ and $f_{c}$ as indicated in Figure 1a

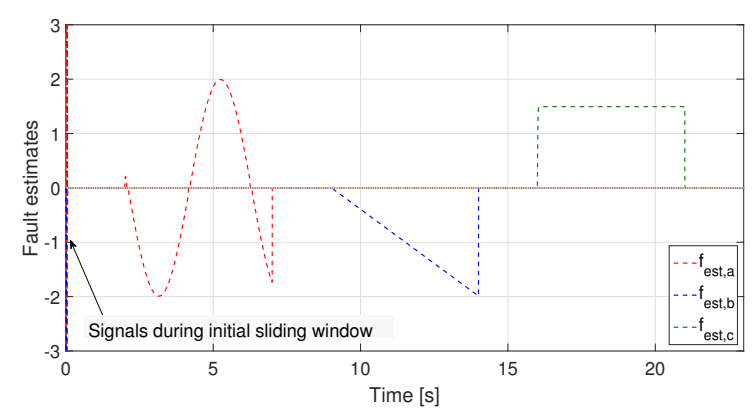

(c) Reconstructed fault estimate signals

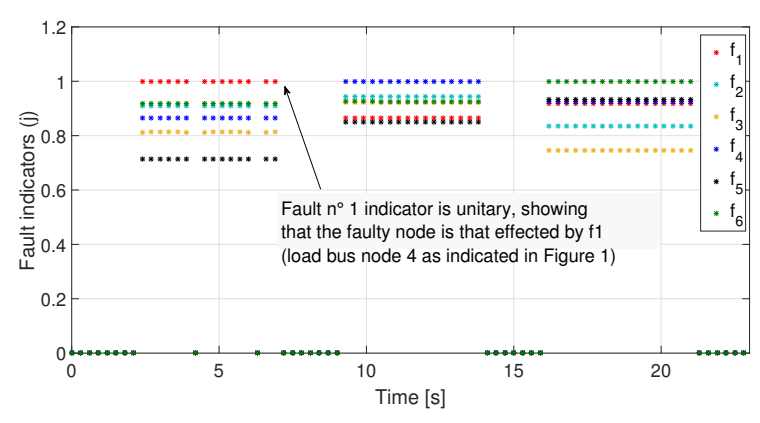

(b) Fault indicators for detection of the channels

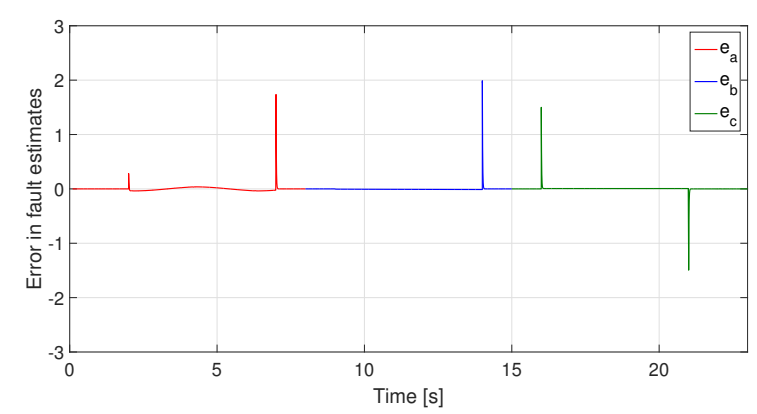

(d) Error in fault reconstruction

Figure 4: Fault reconstruction - 9-bus scenario

where $j$ is the channel in which the failure is identified. As this procedure is applied only when the presence of a fault has been detected, $\left\|\nu_{e q}\right\|>\epsilon$ (assuming that $\Psi_{i} \neq 0$ ), and hence $j$ is well defined.

The final step of the method is fault reconstruction, aimed at reconstructing the shape of the signal $f(t)$; this is obtained through the expression:

$$
|\hat{f}(t)|:=\frac{\left\|\nu_{e q}(t)\right\|}{\left\|\Psi_{j}\right\|}
$$

where $\hat{f}(t)$ is the estimate of the fault $f(t)$.

Remark 4 Consider the system in (6) - (7) with bounded uncertainty on the coefficients of inertia and damping of the generators, $\Delta \mathcal{M}_{g}$ and $\Delta \mathcal{D}_{g}$ respectively. For the observer dynamics in (21), with control gains designed as in (27) - (28), the error dynamics in (31)-(32) remains identical, except for an additional term in (32). Through the analysis of the Lyapunov function in (33) it can be shown that, by an appropriately large choice of the observer gains $k_{1}, \ldots, k_{4}, \dot{V}$ can be rendered negative and second order sliding obtained. The proposed state estimation approach is thus robust with respect to uncertainties on the generator parameters. A similar result holds for uncertainty in the line parameters, modelled as a bounded variation of the Laplacian matrix (5). Both the generator and the line uncertainties have an effect on the fault identification and reconstruction procedure, as they appear in (35). However, it may be possible to analyse the statistical properties of the injection term in order to distinguish between model parameter uncertainties and load altering faults [28].

\section{Simulation Results}

In this section, a set of simulation results, obtained applying the observer design and the fault detection and reconstruction scheme in a Matlab/Simulink environment, will be analysed. The 9-bus and 14-bus IEEE benchmarks are shown in Figs. 1a and $1 \mathrm{~b}$ respectively. Data for these networks is available in the MATPOWER package for Matlab [16]. The 9-bus power network is composed of 3 generator buses $\left(n_{g}=3\right)$ and 6 load buses $\left(n_{l}=6\right)$, whereas the 14-bus power network comprises 5 generator buses $\left(n_{g}=5\right)$ and 9 load buses $\left(n_{l}=9\right)$. The simulations have been run applying the Euler integration method, with a fixed step of $10^{-4}$ seconds.

\subsection{Observer design}

Prior to analysing the results, some details about the super-twisting observer design procedure will be given. The parameter $\eta$ in Theorem 1 has been chosen as $\eta=100$. Taking into account the constraints on the values 


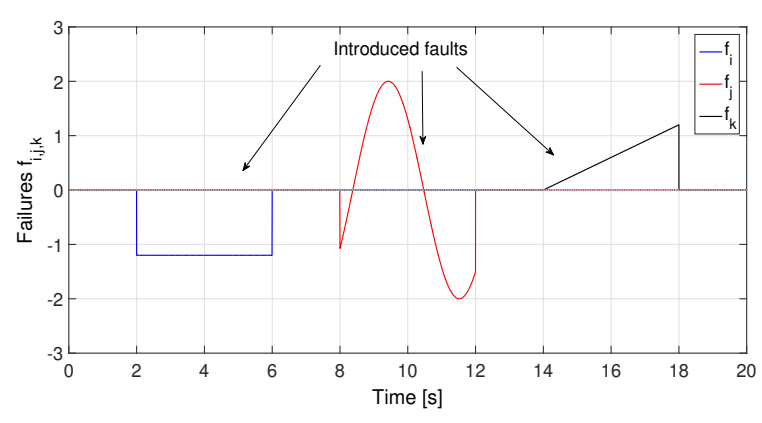

(a) Fault signals $f_{i}, f_{j}$ and $f_{k}$ as indicated in Figure $1 \mathrm{~b}$

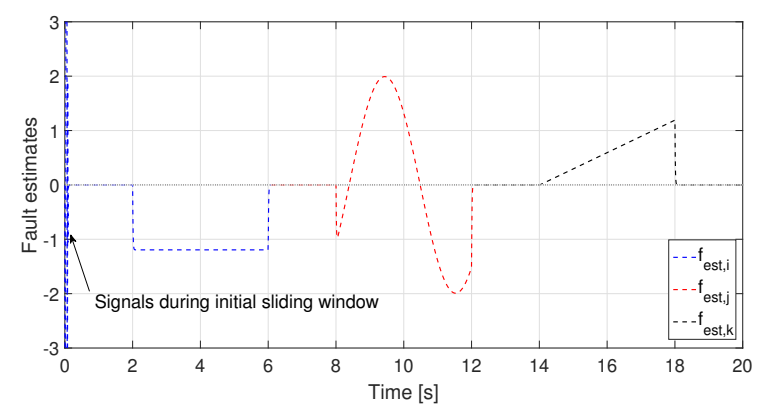

(c) Reconstructed fault estimate signals

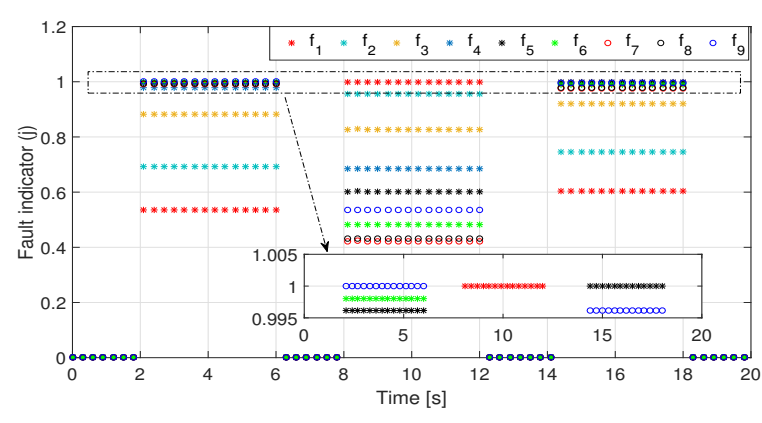

(b) Fault indicators for detection of the channels

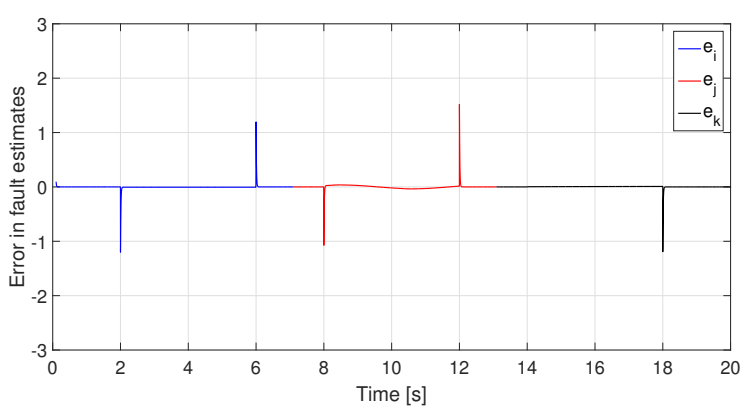

(d) Error in fault reconstruction

Figure 5: Fault reconstruction - 14-bus scenario

of the design variables given in [17], the observer gains $k_{1}, \ldots, k_{4}$ in (27) and (28) have been chosen as given in Table 1. Having satisfied the algebraic constraints in [17] guarantees that $e_{\delta}=\dot{e}_{\delta}=0$ will be obtained in finite time. The designed values, moreover, are conservative, rendering the observer robust enough to analyse both the 9 and 14 bus networks.

\section{$5.2 \quad$ Fault free scenario}

Results from a fault free scenario are first analysed to assess the capability of the observer, designed as described in Section 3, to correctly estimate the system state, exploiting the available measurements. Fig. 2 and Fig. 3 show the results obtained from the analysis of the 9 and the 14 bus networks respectively. The errors in the estimation of the differential states, related to the generators, are shown in Fig. 2a and Fig. 3a, while the estimation errors of the algebraic states, and thus the load phase angle estimations, are shown in Fig. $2 \mathrm{~b}$ and Fig. 3b. The results in Fig. 3, relative to the 14 buses network, have been obtained introducing uncertainty on the generator inertia and damping coefficients. In particular, the coefficients of generators $1,3,5$ have been increased by $15 \%$ from their nominal value, while the coefficients of generators 2,4 have been reduced by $15 \%$. The observer, despite being designed on the basis of the nominal values, is robust with respect to these uncertainties and correctly estimate the full state of the power network model. The multivariable super twisting observer, in fact, forces all the error variables to zero simultaneously in finite time. The convergence to zero happens in approximately $0.06 \mathrm{~s}$ and $0.09 \mathrm{~s}$ for the 9 and the 14 bus networks respectively.

\subsection{Faulty scenario}

Different distinct faults, at different load locations, are considered, as shown in Fig. 1. The position, the time at which they occur and the shape of the introduced faults $\left(f_{a}(t), f_{b}(t), f_{c}(t)\right.$ for the 9 bus network, $f_{i}(t)$, $f_{j}(t), f_{k}(t)$ for the 14 bus network) are described in Table 2. Fig. 4 shows the results of the fault identification and reconstruction procedure in the 9 bus scenario. The faults are represented in Fig. 4a. Fig. 4b shows the result of the fault identification phase: the fault indicators are different from zero only in the time intervals when the fault identification procedure is active, that is when condition (36), with $\epsilon=1$, is satisfied. In each interval of operation, the unitary indicator determines the faulty channel: for instance, between 2 and 7 seconds the indicator $f_{1}$ is unitary. This means that the fault is located in load bus number 1 , which, considering the presence of 3 generators in the network, is bus number 4. As highlighted in Fig. 1a, $f_{a}$ affects bus 4; this confirms the efficacy of the method. The results of the fault reconstruction procedure are shown in Fig. 4c, 
Table 3: Observer's performances

\begin{tabular}{|c|c|c|}
\hline 9 - bus & 0.0521 & 0.0329 \\
\hline 14 - bus & 0.0460 & 0.0380 \\
\hline
\end{tabular}

while the fault reconstruction error is shown in Fig. 4d. The spikes in the error are due to abrupt changes in the values of the estimated variables. These, however, are almost immediately reduced to zero (the amplitude is of the order of $1 \%$ of the amplitude of the introduced fault).

A similar set of results for the 14 bus network is shown in Fig. 5. The faults are shown in Fig. 5a. Fig. 5b shows the results of the fault detection procedure: again, during each fault interval, the unitary fault indicator is the correct one. Between 14 and 18 seconds, for example, the unitary fault indicator is $f_{5}$ : this corresponds to a fault in the fifth load bus, which is bus 10 . Fig. $5 \mathrm{c}$ shows the results of the fault reconstruction procedure and Fig. 5d shows the fault reconstruction error.

\subsection{Summary}

In order to compare the results obtained with the multivariable super twisting sliding mode observer when considering the 9 bus and the 14 bus network, the Root Mean Square Error (RMSE) value has been used as a performance index. In particular, considering the fault free simulations presented in Section 5.2, the RMSE related to the differential states has been computed as:

$$
R M S E_{d}=\frac{1}{n_{d}} \sum_{i=1}^{n_{d}} \sqrt{\frac{1}{k} \sum_{j=1}^{k}\left(e_{i j}\right)^{2}}
$$

where $k$ is the number of samples in the simulation interval, $n_{d}$ is the number of differential states and $e_{i j}$ is the estimation error of the $i$-th differential variable at sampling time $j$. The RMSE value relative to the algebraic variables $\left(R M S E_{a}\right)$ has been computed accordingly. The results obtained, relative to the fault free simulations, are shown in Table 3. The values of the Root Mean Square Error in Table 3 indicate a high level of performance when estimating the state of the differential algebraic system of equations. This, together with a high level of precision in the identification of the faulty channel and in the estimation of the faults, indicates that the scheme proposed in this paper is highly satisfactory.

\section{Conclusion}

A fault reconstruction scheme for a class of DAEs representing a power network has been proposed, based on a multivariable super-twisting sliding mode observer. The approach only requires that a subset of the differential states (the phase angles of the generators) is measured. Using these measurements the complete state of the model can be estimated; moreover, an approach to detect, isolate and reconstruct a single failure in any of the algebraic states (the phase angle of the load bus tension) has been developed. The effectiveness of the scheme has been assessed considering two IEEE benchmarks. The scheme has been tested by introducing faults in the channels of the load bus tensions. The simulation results have been analysed and discussed, showing the high level of effectiveness of the proposed observer based approach. As a first extension of the present work, the possibility to detect, identify and reconstruct simultaneous failures, occurring at different buses, could be considered. In addition, a complete analysis of the model parameter uncertainties could be performed. An additional layer could be added in the fault detection procedure in order to distinguish the effect of load altering faults from the effect of parameters uncertainties. Finally, as the proposed observer is centralized, the possibility to design distributed observers, in order to render the approach decentralized and scalable, could be considered.

\section{References}

[1] Darouach, M:, 'Observers and observer-based control for descriptor systems revisited', IEEE Transactions on Automatic Control, 2014, 59, (5), pp. 1367-1373

[2] Kundur, P., Balu, N.J., Lauby, M.G.:'Power system stability and control', (McGraw- Hill New York, 1994) 
[3] Scholtz, E.: 'Observer based monitors and distributed wave controllers for electromechanical disturbances in power systems', Massachusetts Institute of Technology, 2004

[4] Pasqualetti, F., Bicchi, A., Bullo, F.: 'A graph-theoretical characterization of power network vulnerabilities', Proc. of American Control Conference, San Francisco, CA, 2011, pp. 3918-3923

[5] Muller, P., Hou, M.: 'On the observer design for descriptor systems', IEEE Transactions on Automatic Control, 1993, 38, (11), pp. 1666-1671

[6] Tarraf, D.C., Asada, H.H.: 'A sliding observer for differential-algebraic (DAE) systems', Proc. of American Control Conference, Arlington, VA, 2001, pp. 4338-4343

[7] Scholtz, E., Lesieutre, B.C.: 'Graphical observer design suitable for large-scale DAE power systems', Proc. of 47th IEEE Conference on Decision and Control, Cancun, Messico, 2008, pp. 2955-2960

[8] Xu, S., Lam, J., Zou, Y.: ' $\mathrm{H}_{\infty}$ filtering for singular systems', IEEE Transactions on Automatic Control, 2003, 48, (12), pp. 2217-2222

[9] Yeu, T.K., Kawaji, S.: 'Sliding mode observer based fault detection and isolation in descriptor systems', Proc. of American Control Conference, Anchorage, Alaska, 2002, pp. 4543-4548

[10] Jinyong, Y., Zhiyuan, L.: 'Fault reconstruction based on sliding mode observer for linear descriptor systems', Proc. of 7th Asian Control Conference, Hong Kong, China, 2009, pp. 1132-1137

[11] Chalanga, A., Kamal, S., Fridman, L.M. et al.: 'Implementation of super-twisting control: super-twisting and higher order sliding-mode observer-based approaches', IEEE Transactions on Industrial Electronics, 2016, 63, (6), pp. 3677-3685

[12] Basin, M.V., Ramirez, P.C.R.: 'A supertwisting algorithm for systems of dimension more than one', IEEE Transactions on Industrial Electronics, 2014, 11, (61), pp. 6472-6480

[13] Manandhar, K., Cao, X., Hu, F. et al.: 'Detection of faults and attacks including false data injection attack in smart grid using Kalman filter', IEEE Transactions on Control of Network Systems, 2014, 1, (4), pp. 370-379

[14] Van Gorp, J., Defoort, M., Djemai, M. et al.: 'Fault detection based on higher-order sliding mode observer for a class of switched linear systems', IET Control Theory $\&$ Applications, 2015, 9, (15), pp. 2249-2256

[15] Liu, J., Vazquez, S., Wu, L. et al.: 'Extended state observer-based sliding-mode control for three-phase power converters', IEEE Transactions on Industrial Electronics, 2017, 64, (1), pp. 22-31

[16] Zimmerman, R.D., Murillo Sanchez, C.E.: 'Matpower 5.1-User's Manual', Power Systems Engineering Research Centre (PSERC), 2015

[17] Nagesh, I., Edwards, C.: 'A multivariable super-twisting sliding mode approach', Automatica, 2014, 50, (3), pp. 984-988

[18] Pasqualetti, F., Dörfler, F., Bullo, F.: 'Cyber-physical attacks in power networks: Models, fundamental limitations and monitor design', Proc. of 50th IEEE Conference on Decision and Control and European Control Conference, Orlando, FL, 2011, pp. 2195-2201

[19] Shames, I., Teixeira, A., Sandberg, H. et al.: 'Distributed fault detection for interconnected second-order systems with applications to power networks', First Workshop on Secure Control Systems, Stockholm, Sweden, 2010

[20] Stott, B., Alsaç, O.: 'Fast decoupled load flow', IEEE Transactions on Power Apparatus and Systems, 1974, 3, pp. 859-869

[21] Van Amerongen, R.A.: 'A general-purpose version of the fast decoupled load flow', IEEE Transactions on Power Systems, 1989, 4, (2), pp. 760-770

[22] Dai, L.: 'Singular control systems', (Springer Berlin Heidelberg, 1989)

[23] Davila, J., Fridman, L., Levant, A. et al.: 'Second-order sliding-mode observer for mechanical systems', IEEE Transactions on Automatic Control, 2005, 50, (11), pp. 1785-1789 
[24] Shtessel, Y.B., Moreno, J.A., Plestan, F. et al.: 'Super-twisting adaptive sliding mode control: A Lyapunov design', Proc. of 49th IEEE Conference on Decision and Control, Atlanta, GA, 2010, pp. 5109-5113

[25] Yan, X.G., Edwards, C.: 'Adaptive sliding-mode-observer-based fault reconstruction for nonlinear systems with parametric uncertainties', IEEE Transactions on Industrial Electronics, 2008, 55, (11), pp. 4029-4036

[26] Edwards, C., Spurgeon, S.K., Patton, R.J.: 'Sliding mode observers for fault detection and isolation', Automatica, 2000, 36, (4), pp. 541-553

[27] Campos Delgado, D.U., Espinoza Trejo, D.R.: 'An observer-based diagnosis scheme for single and simultaneous open-switch faults in induction motor drives', IEEE Transactions on Industrial Electronics, 2011, 58, (2), pp. 671-679

[28] Pasqualetti, F., Dörfler, F., Bullo, F.: 'Attack detection and identification in cyber-physical systems', IEEE Transactions on Automatic Control, 2013, 58, (11), pp. 2715-2729 